\title{
Geometría del ancho de banda de un canal de comunicación
}

\section{Bandwidth geometry of a communication channel}

Deysi Margoth Guanga Chunata. ${ }^{1}$, Oswaldo Martínez Guashima. ${ }^{2}$, Mercedes Leticia Lara Freire. ${ }^{3}$ \& Omar Vinicio Galarza Barrionuevo. ${ }^{4}$

Abstract.

DOI: https://doi.org/10.33262/cienciadigital.v4i2.1207

The objective of this work is to show the results of a study carried out with a group of 50 Telecommunications engineering students to whom the cognitive construction of geometric elements in the determination of the bandwidth of a channel, it was applied as a learning test. Mathematical instruction in the epistemic and cognitive dimensions. In this search work were implement a didactic strategy and a construction methodology, designed on geometric elements to reason about conditional probability. The productions analyzed before and after the instruction. The purpose was to contribute to the construction of the meaning of the formula programmed in the software or firmware of a network interface, by promoting the ability to carry out the cognitive construction of the communication channel and its behavior in a work setting. Students who developed this skill showed less difficulty in their resolutions and came up with new ideas on optimizing.

Keywords: Geometry, Communication Systems, Bandwidth, Coding

${ }^{1}$ Escuela Superior Politécnica de Chimborazo, Facultad de Informática y Electrónica. Riobamba, Ecuador. deysi.guanga@espoch.edu.ec

${ }^{2}$ Escuela Superior Politécnica de Chimborazo, Facultad de Informática y Electrónica. Riobamba, Ecuador. geovanny.martinez@espoch.edu.ec

${ }^{3}$ Escuela Superior Politécnica de Chimborazo, Facultad de Informática y Electrónica. Riobamba, Ecuador. leticia.lara@espoch.edu.ec

4 Universidad de la Fuerzas Armadas, Departamento de Ciencias Exactas. Sangolquí, Ecuador. ovglarza@espe.edu.ec 


\section{Resumen.}

Este trabajo tiene por objeto mostrar los resultados de un estudio planteado con un grupo de 50 estudiantes de ingeniería en Telecomunicaciones a quienes se les aplicó la construcción cognitiva de los elementos geométricos en la determinación del ancho de banda de un canal como una prueba de aprendizaje, la instrucción Matemática en las dimensiones epistémica y cognitiva. Se implementó una estrategia didáctica y una metodología de construcción, diseñada sobre los elementos geométricos para razonar sobre probabilidad condicional. Las producciones se analizaron antes y después de la instrucción. El propósito era contribuir a la construcción del significado de la fórmula programada en el software o firmware de una interfaz de red, al favorecer la habilidad de efectuar la construcción cognitiva del canal de comunicación y su comportamiento en un escenario de trabajo. Los estudiantes que desarrollaron esta habilidad, mostraron menores dificultades en sus resoluciones y plantearon nuevas ideas sobre optimización del canal de comunicación.

Palabras claves: Geometría, Sistemas de comunicación, Ancho de banda, Codificación

\section{Introducción.}

En la actualidad, las categorías más utilizadas en la teoría de la comunicación y sus aplicaciones han sido la definición de límites fundamentales en la velocidad y confiabilidad de la transferencia de información, la capacidad del canal y la relación señal / ruido como un indicador determinante de la calidad de comunicación prevista. Sin lugar a dudas, la principal categoría de la teoría moderna de la información es el concepto de ancho de banda de un canal. (Facchini, 2020)

En su interpretación, el ancho de banda se mide como la cantidad de datos que se pueden transferir entre dos puntos de una red en un tiempo específico. Normalmente, el ancho de banda se mide en bits por segundo (bps) y se expresa como una tasa de bits. La medición del ancho de banda se realiza normalmente mediante software o firmware en una interfaz de red. Normalmente, para medir el ancho de banda, se calcula la cantidad total de tráfico enviado y recibido en un período de tiempo específico. (Amado, 2019)

La capacidad del canal se expresó en términos estadísticos a través de la característica matemática introducida de la distribución de probabilidad conjunta de dos variables aleatorias, denominada cantidad de información. La cantidad máxima de información en la señal en la salida del canal con respecto a la señal en su entrada, donde el máximo se toma sobre todas las posibles distribuciones de probabilidad de la señal en la entrada. La cantidad de información, a su vez, se expresa a través de otra cantidad que se ha utilizado durante 
mucho tiempo en termodinámica: la entropía y representa la diferencia entre la entropía de una señal en la salida del canal y su entropía condicional si se conoce la señal en la entrada.

Pese a los logros innegables en la teoría de la información, la falta de constructivismo práctico en varias formulaciones de teoremas y los problemas de desarrollo, el progreso tecnológico visible de todos los tipos de servicios de comunicación es prácticamente incapaz de ocultar el hecho de la ausencia de un aumento significativo en la eficiencia específica de los equipos de telecomunicaciones (Castro-Ladino, 2019).

Los protocolos del sistema de transferencia de información de canal y capa física (SPI) son, en gran medida, costosos. Los códigos resistentes a interferencias, cuya historia de estudios teóricos y experimentales se han llevado a cabo durante más de 70 años, se usan en la práctica en el modo de corrección de errores. La razón de esto no es solo la complejidad computacional de construir y decodificar estructuras voluminosas en canales de alta velocidad, sino también la inaceptabilidad de una probabilidad residual bastante sustancial de decodificación (corrección) con un error en la transmisión de datos y textos de programas de computadora.

El desarrollo de la tecnología de las comunicaciones es de naturaleza puramente extensa. La ganancia de rendimiento se logra casi exclusivamente al mejorar la base tecnológica para la implementación de dispositivos transceptores, así como al expandir la banda de frecuencia y la potencia del transmisor (que, de hecho, determina la definición matemática del rendimiento). Esto tiene ciertas consecuencias negativas en los aspectos morales, materiales y ambientales. El problema de la compatibilidad electromagnética es cada vez más importante. Los rangos tradicionales de radiofrecuencias sobrecargados y un pequeño ancho de banda de las líneas de comunicación metálica forzaron el uso del rango óptico, cuyas posibilidades tampoco son ilimitadas y requieren de dispositivos de acondicionamiento de señal adicionales.

Las tecnologías móviles, que van desde 2G, LTE y hasta el prometedor 5G, sufre cambios drásticos, con la excepción de modificaciones extensas. La órbita geoestacionaria de los satélites de comunicación se acerca a la máxima saturación con objetos espaciales. La tasa de aumento en la demanda de servicios de comunicación excede la tasa de aumento en la productividad de SPI. Todo esto, por supuesto, es evidencia de los problemas obvios de la teoría y la práctica de construir sistemas de transferencia de información (Vesga Ferreira, 2019).

\section{Construcción Cognitiva}

Los elementos matemáticos son necesarios y se evidencian como prerrequisitos para los estudiantes de ingeniería en Telecomunicaciones, por otra parte. La Teoría APOE (Acrónimo de Acción, Proceso, Objeto y Esquema) es una teoría constructivista que toma como marco de referencia las ideas de Piaget respecto al desarrollo del conocimiento, fundamentalmente 
rescatando el concepto de abstracción reflexiva y el concepto de esquema. Dubinsky, quien desarrolla esta teoría, extiende el análisis cognitivo de conceptos matemáticos que se estudian de un nivel escolar, a un nivel de educación superior (García-Martinez, 2017)

En la teoría APOE, las estructuras mentales que describen el proceso de construcción de un fragmento del conocimiento matemático constituido en cuatro categorías: acción, proceso, objeto y esquema. Conforme un individuo reflexiona y trabaja para comprender uno o varios conceptos matemáticos pone en juego mecanismos mentales: interiorización, coordinación, encapsulación y reversión, que son considerados casos particulares de la abstracción reflexiva (GODINO, Indicadores de la idoneidad didáctica de procesos de enseñanza y aprendizaje de las matemáticas., 2011)

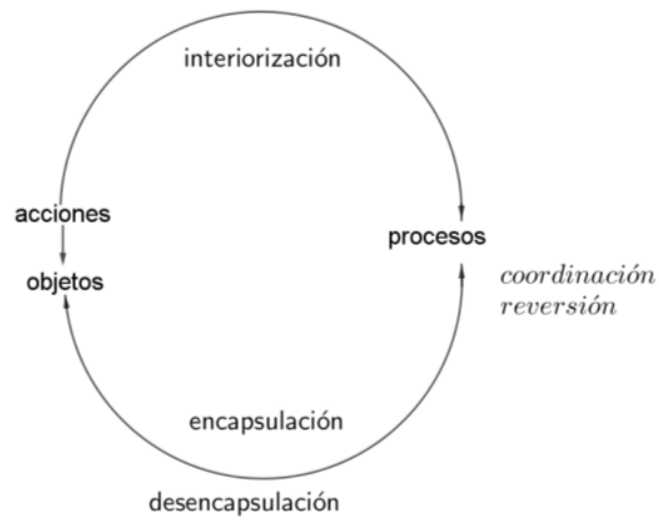

Figura 1: Estructuras y mecanismos mentales para construir conceptos

Fuente: Elaboración propia

Al considerar un objeto matemático se realizan acciones sobre objetos previamente construidos, relacionándolos con otros conceptos matemáticos que se toman como base para la construcción del nuevo. Las acciones se caracterizan por ser transformaciones que se realizan paso a paso, obedeciendo a estímulos que son o se perciben como externos (Dubinsky, 1996)

Un estudiante ha interiorizado una acción en un proceso si puede realizar una operación interna que hace (o imagina) esencialmente la misma transformación enteramente en su mente, sin necesariamente realizar todos los pasos específicos. (Arnon, 2014)

La teoría APOE proporciona un ciclo de investigación compuesto por tres componentes: el análisis teórico, el diseño y aplicación de instrumentos y el análisis y verificación de datos, el cual ha sido utilizado con éxito por el Grupo Rumec y otros investigadores (GarcíaMartinez, 2017) 
Sobre la primera componente del método surge la interrogante ¿Cómo representar geométricamente el ancho de banda de un canal? Puede ser que la representación corresponda a una manifestación de algo más profundo, por un lado, definir el espacio que limita el canal de información y la forma como transmite datos o señales no sólo corresponde a una lista de números.

\section{Metodología.}

Al diseñar esta propuesta didáctica se considera en primer lugar, los conocimientos previos de los alumnos, sus experiencias y el contexto en el que están inmersos. Dado que la asignatura Redes de Comunicación es común a muchas carreras de ingeniería en telecomunicaciones, el profesor hace preguntas a sus alumnos para involucrarlos y despertar su interés, en este caso se pregunta a cómo calcular el ancho de banda de un canal de comunicación.

Una forma de determinar el ancho de banda basado en la construcción geométrica multidimensional de la señal y el espacio de interferencia, presentado en la aproximación "plana" en la Figura. 2. Cualquier implementación de una señal aleatoria continua de duración $\boldsymbol{T}$, cuyo espectro está limitado por la frecuencia $\boldsymbol{F}$, con $n=2 F T$ está representada por un punto en el espacio dimensional. Considerando un sistema de transmisión en óptimas condiciones estos puntos denominados $S_{i}$ se distribuyen equitativamente dentro de una hiperesfera con un radio determinado por la potencia de señal promedio y la dimensión espacial:

$$
r_{S} \approx \sqrt{n S}
$$

En volumen:

$$
V_{s} \approx \frac{\sqrt{\pi}^{n}}{\Gamma\left(\frac{n}{2}+1\right)}(n S)^{n}
$$

Donde $\Gamma\left(\frac{n}{2}+1\right)$ es la función gamma para una distribución uniforme de los puntos de señal, puede que puede ubicarse aleatoriamente en las n coordenadas: variables aleatorias con media cero y una dispersión igual a S. Con un aumento ilimitado en la dimensión del espacio $\boldsymbol{n}$, la distribución de puntos se acercará monótonamente a la distribución uniforme. Esta propiedad asintótica de uniformidad es la base para construir códigos aleatorios, Una implementación aleatoria de una señal es una forma de canal de la palabra de código de un código aleatorio y se puede obtener de una de dos maneras:

$$
S(t)=\sum_{i=0}^{2 F T-1} S_{i} \frac{\sin (2 \pi F(t-i \cdot \Delta t))}{2 \pi F(t-i \cdot \Delta t)}, \quad \Delta t=\frac{1}{2 F}
$$


Que representa la descomposición aleatoria en base a funciones $\boldsymbol{s i n}$ de referencia y tiene un espectro de frecuencia $\boldsymbol{F}$ continuo.

$$
S(t)=\sum_{i=1}^{2 F T}\left\{S_{2(i-1)} \sin \left(2 \pi F \frac{i}{T}\right)+S_{2(i-1)+1} \cos \left(2 \pi F \frac{i}{T}\right)\right\}
$$

Que representa la expansión de Fourier aplicado en el armónico ortogonal en el intervalo T, con implementación $S(t)$, t es periódico en $\mathrm{T}$ y, si se continúa indefinidamente su repetición, tendrá un espectro discreto limitado anteriormente por un valor $\mathrm{F}$, cuyas mediciones distintas de cero se encuentran en incrementos de $\frac{1}{T}$ en frecuencia.

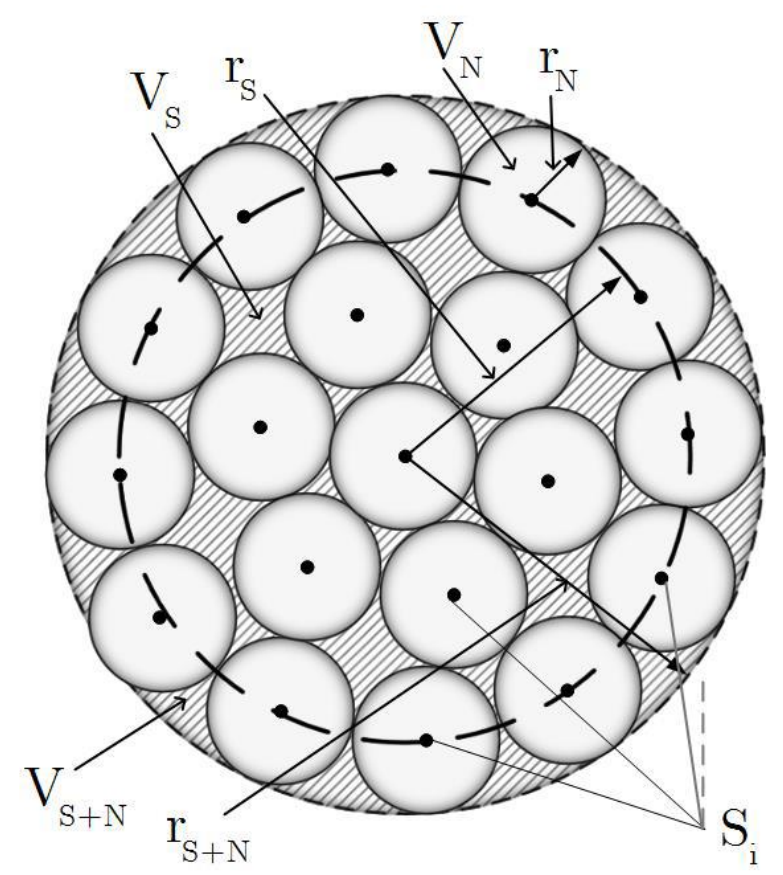

Figura 2. Representación geométrica del espacio del sistema de transmisión de información.

Fuente: Elaboración Propia

Ambos métodos las ecuaciones (3) y (4) se pueden usar para describir el rendimiento con la ayuda de la codificación dada por Shannon. Se aplican $m=2^{k}$ muestras de ruido, cada duración T. Con valores de 0 a $m-1$. En el transmisor, las secuencias de mensajes se dividen en grupos de $\mathrm{k}$ dígitos binarios y para cada grupo la muestra de ruido correspondiente se transmite como una señal. En el extremo receptor, se conocen estas $\boldsymbol{m}$ muestras, y la señal realmente recibida (distorsionada por el ruido) se compara con cada una de ellas. La muestra que tiene la desviación estándar más pequeña de la señal recibida se toma como la señal transmitida, a partir de la cual se restaura el número binario correspondiente. Esta técnica es equivalente a elegir la señal más probable. 
Las ecuaciones (3), (4) junto con la descripción anterior muestran el proceso de construcción y decodificación de un código aleatorio. La decodificación se lleva a cabo de acuerdo con la regla, que ahora se llama tradicionalmente "Regla de máxima verosimilitud" (PMP). Con un aumento ilimitado en la longitud del bloque de código (aumento sincrónico en los parámetros k y $n=2 F T$. Si la interferencia no es demasiado grande, el error al determinar la palabra de código recibida puede hacerse arbitrariamente pequeño. Por lo tanto, en definición geométrica, el rendimiento es la velocidad de código aleatorio más alta posible al decodificarla en el PMP y asegurando una inseguridad arbitrariamente baja.

En la interpretación geométrica del mejor código (Figura. 2) en la salida de los puntos del canal $S_{i}, \quad i \in[0, m-1]$ corresponde a las palabras de código transmitidas, bajo la influencia de un gaussiano. Las interferencias se desplazan dentro de esferas de incertidumbre con un radio:

$$
r_{N} \approx \sqrt{n N}
$$

y volumen:

$$
V_{n}=\frac{\sqrt{\pi}^{n}}{\Gamma\left(\frac{n}{2}+1\right)}(\sqrt{n N})^{n}
$$

De acuerdo con la manifestación de la ley de los grandes números con $\boldsymbol{n}$ creciente, la probabilidad de encontrar puntos desplazados fuera de una esfera con un radio $r_{N}+\frac{\varepsilon}{\sqrt{n}}$ tiende a cero ( $\varepsilon$ valor arbitrariamente pequeño). Las esferas de incertidumbre adquieren una forma más definida. Shannon los comparó con las bolas de billar, dado que las señales de palabra de código y la interferencia son independientes entre sí, el radio total del espacio hiperesférico que contiene $\mathbf{m}$ esferas de incertidumbre se caracteriza por el radio y el volumen:

$$
\begin{gathered}
, r_{S+N} \approx \sqrt{n(S+N)} \\
V_{S+N} \approx \frac{\sqrt{\pi}^{n}}{\Gamma\left(\frac{n}{2}+1\right)}(\sqrt{n(S+N})^{n}
\end{gathered}
$$

En $n \rightarrow \infty$ determina el número máximo de esferas disjuntas que se pueden empaquetar en el volumen $V_{S+N}$ de tal manera que entre ellos, prácticamente no hay espacios vacíos:

$$
m_{C}=\frac{V_{S+N}}{V_{N}}=\sqrt{\frac{S+N}{N}}^{n}=\sqrt{\frac{S+N}{N}}^{2 F T}
$$


De acuerdo con la construcción de palabras de código las ecuaciones (3) y (4) , $n=2 F T$ determinan la dimensión del espacio de código geométrico. La ecuación (9) y el promedio en el tiempo T se obtiene la máxima velocidad de código alcanzable, o (como afirma la teoría moderna de la información) el ancho de banda del canal:

$$
C=\frac{1}{T} \log m_{C}=F \log \left(\frac{S+N}{N}\right)
$$

C revela el resultado de la tasa de transferencia de información máxima alcanzable en un canal con ruido aditivo con una confiabilidad arbitrariamente pequeña. Sin embargo, debe tenerse en cuenta que en la lógica de la derivación de la ecuación (10), el valor C es el límite de velocidad del mejor código, usando la regla de máxima verosimilitud al decodificar. Si esto no fuera así y el receptor no tuviera que almacenar en la memoria muestras de segmentos de implementaciones de señal para su uso en comparaciones de PMF, entonces para $N>S$ sería suficiente cambiar a recepción de interferencia, luego para compensarlo en la mezcla de salida del canal. Puede recurrir que el significado físico y matemático del ancho de banda y encontrar que el valor $\mathrm{C}$ en los teoremas probados es precisamente el límite superior de las velocidades de transmisión para códigos en un canal gaussiano usando PMP, pero no para un canal gaussiano con cualquier método de transmisión y procesamiento de señales.

En las opiniones predominantes de la teoría de la información, no hay diferencia entre los dos conceptos, ya que la presencia de un codificador y un decodificador de canal es obligatoria por defecto en el esquema SPI introducido por Shannon.

La posibilidad de construir un SPI efectivo que no use codificación no está permitido, esto contradice drásticamente la observación práctica observada en la introducción de que los códigos de corrección de errores en el modo de corrección de. Con confianza se puede decir que el valor $\mathrm{C}$ representa la velocidad máxima de transferencia de información a través del canal de ruido aditivo o la velocidad máxima de transmisión de información en el canal cuando se utiliza la codificación y las reglas para decodificar PMP (bajo la condición de una confiabilidad arbitrariamente baja).

\section{Resultados.}

Como parte de la idoneidad cognitiva, y para la componente Aprendizaje, es necesario partir de las configuraciones cognitivas de los alumnos antes de la metodología de resolución y después del proceso de instrucción.

Hubo un mayor número de alumnos que establecieron correctamente más funciones cognitivas sobre la medición del ancho de banda sin utilizar software lo cual se evidencia en la instancia de evaluación, aumentando la frecuencia de los puntajes más altos (puntajes obtenidos en los ensayos experimentales y comparación con simulaciones en el laboratorio), como se muestra en la Tabla 1. 
Tabla 1 Resumen de la aplicación del método en dos grupos

\begin{tabular}{|c|c|}
\hline Fuente & $\begin{array}{lll}\text { - Caso } 1 & \text { Caso } 2 \\
\text { - } 20 \text { Estudiantes de ingeniería en } & \bullet \quad 30 \text { estudiantes de Ingeniería en } \\
\text { Telecomunicaciones. } & \text { Telecomunicaciones. }\end{array}$ \\
\hline Técnica & $\begin{array}{l}\text { - Aplicación del modelo cognitivo de la geometría del ancho de banda } \\
\text { - } \quad \text { Definición directa y textual de la geometría del ancho de banda }\end{array}$ \\
\hline $\begin{array}{l}\text { Análisis } \\
\text { de los } \\
\text { datos }\end{array}$ & $\begin{array}{l}\text { - Caso 1, Caso } 2 \\
\text { - Del grupo de } 50 \text { estudiantes se aplicó un conjunto de problemas orientados al } \\
\text { cálculo del ancho de banda y posibles medidas de optimización y mejora con } \\
\text { elementos varios, } 25 \text { de estudiantes completaron la tarea establecida con } \\
\text { sugerencias de mejora u optimización del canal de comunicación, los } 25 \\
\text { estudiantes corresponden al grupo en el que se aplicó el método. }\end{array}$ \\
\hline
\end{tabular}

Fuente: Elaboración propia

\section{Conclusiones.}

- La imposibilidad práctica de determinar analíticamente el rendimiento de dichos modelos.

- La definición matemática describe correctamente la cantidad de rendimiento de cualquier canal continuo en el que el ruido es un proceso aleatorio estacionario.

- El tipo de distribución de ruido no afecta la cantidad de ancho de banda, que está completamente determinada por la relación señal-ruido y la banda de frecuencia.

- Las diferentes distribuciones de ruido aparecen solo en un cambio en la velocidad de acercamiento al rendimiento y la capacidad de aumentar la duración de segmentos de secuencias de ruido de un código aleatorio.

- La definición geométrica correcta del ancho de banda describe su naturaleza física como la velocidad máxima de transmisión de información en el canal con cualquier tipo de ruido aditivo cuando se utiliza codificación / decodificación de canales y aplicación de la regla de máxima verosimilitud para la decodificación.

\section{Referencias Bibliográficas.}

Alfonso, A. G. (2012). Comprensión lectora y concepciones de estudiantes universitarios sobre enunciados matemáticos. Instituto de Estudios Superiores en Educación, , (17), 2-23.

Amado, J. P.-C. (2019). SOLUTION OF A P AND S WAVE PROPAGATION MODEL USING HIGH PERFORMANCE COMPUTATION. CT\&amp; F - Ciencia, Tecnología y Futuro, 9(1); 119-130. 
Arnon, I. C. (2014). A framework for research and curriculum development in mathematics education. Springer. APOS Theory, doi: 10.1007/978-1-4614-7966-6. Obtenido de A framework for research and curriculum development in mathematics education.

Castro-Ladino, J. R.-B. (2019). Aplicación de metamateriales para estimar índices de refracción. Revista de la Academia Colombiana de Ciencias Exactas, Físicas y Naturales, 43(167); 193198.

DÍAZ, C. D. (2014). Razonamiento sobre Probabilidad Condicional e implicaciones para la enseñanza de la Estadística. Andalucía: Epsilon,.

Dorier, J. L. (2002). The teaching and learning of mathematics at university level. New ICMI Study Series, 7(3), 255-273.

Dubinsky, E. (1996). Aplicación de la perspectiva piagetiana a la educación matemática universitaria. Educación Matemática, , 8(3), 24-41.

Facchini, ,. H. (2020). Evaluación de métricas del comportamiento del tráfico de video en una red experimental multidifusión. Enfoque UTE; , 11(1); 15-27.

FONT, V., GODINO, J., \& D'AMORE, B. (24 de 10 de 2012). Enfoque Ontosemiótico de las representaciones en Educación Matemática. v. 27, n. 2, p. 3-9. 2007. Obtenido de For the learning of mathematics, Montreal,: <http://www.ugr.es/ jgodino/menuing.htm>. Acceso en: 24 oct. 2012.

García-Martinez, I. \&. (2017). The basis step in the construction of the principle of mathematical induction based on APOS theory. The Journal of Mathematical Behavior, 46, 123-143.

GODINO, J. (2011). Indicadores de la idoneidad didáctica de procesos de enseñanza y aprendizaje de las matemáticas. CONFERENCIA INTERAMERICANA DE EDUCACIÓN MATEMÁTICA, (págs. p. 1-20.). CONFERENCIA INTERAMERICANA DE EDUCACIÓN MATEMÁTICA,.

GODINO, J., \& BATANERO, C. S. (24 de 10 de 2012). Recherches en Didactique des Mathématiques. Obtenido de Grenoble, : <http://www.ugr.es/ jgodino/menuing.htm>.

POLLATESK, A., WELL, A., KONOLD, C., HARDIMAN, P., \& COBB, G. (1987). Understanding Conditional Probabilities. Organizational, Behavior and Human Decision Processes,, n. 40, p. 255-269.

Rafael-Valdivia, G. (2019). Modelo compacto con capacidad de predicción de parámetros físicos para amplificadores de RF. Revista Facultad de Ingeniería, 28(51); 73-87.

Vesga Ferreira, J. C. (2019). Optimización del ancho de banda en redes BPL usando las técnicas nucleolus y max-min fairness. Revista Ingenierías Universidad de Medellín, 18(34); 165 180. 


\section{PARA CITAR EL ARTÍCULO INDEXADO.}

Guanga Chunata, D. M., Martínez Guashima, O., Lara Freire, M. L., \& Galarza Barrionuevo, O. V. (2020). Geometría del ancho de banda de un canal de comunicación. Ciencia Digital, 4(2), 77-87. https://doi.org/10.33262/cienciadigital.v4i2.1207

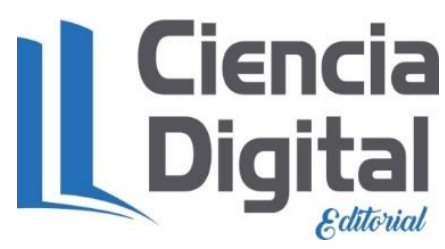

El artículo que se publica es de exclusiva responsabilidad de los autores y no necesariamente reflejan el pensamiento de la Revista Ciencia Digital.

El artículo queda en propiedad de la revista y, por tanto, su publicación parcial y/o total en otro medio tiene que ser autorizado por el director de la Revista Ciencia Digital.
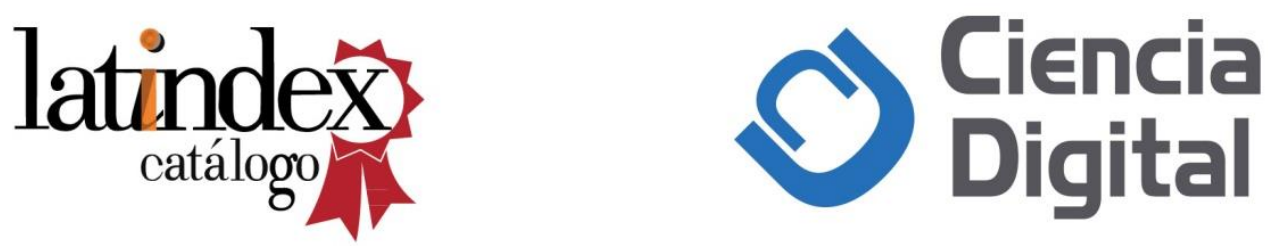